\title{
Uncertainties on Modal Parameters by Operational Modal Analysis
}

\author{
VIET HUNG VU and MARC THOMAS \\ École de technologie supérieure (ÉTS), 1100 Notre Dame Ouest, Montréal, Québec, H3C 1K3, Canada \\ marc.thomas@etsmtl.ca
}

\section{Résumé :}

Lorsqu'on fait une analyse modale opérationnelle sur des structures en fonctionnement, sans connaître les forces de perturbation, ni le nombre de fréquences importantes qui excitent le système mécanique, on doit se poser la question sur la validité de l'estimation des paramètres modaux (notamment le taux d'amortissement) et sur la précision des résultats obtenus. Cet article présente une méthode qui permet d'évaluer l'incertitude des paramètres modaux (fréquences et taux d'amortissement). L'analyse modale opérationnelle est réalisée à partir d'un modèle vecteur autorégressif (VAR) (à plusieurs canaux de mesure) pour le traitement des signaux temporels et l'identification modale. Comme l'incertitude des paramètres modaux (fréquence et taux d'amortissement) diminue en fonction de l'ordre considéré du système, le calcul de l'incertitude permet également de définir un critère objectif pour sélectionner l'ordre optimal du modèle, en fonction d'une marge d'erreur acceptable de prédiction. Il faut toutefois mentionner que l'identification des fréquences naturelles est plus aisée que celle du taux d'amortissement et demande un ordre de calcul moins important. La simulation numérique et la mesure vibratoire réelle sur une plaque métallique montrent l'efficacité et la faisabilité de la méthode développée.

\begin{abstract}
:
When operational modal analysis (OMA) is conducted on operating machines or structures without knowing excitations and perturbations neither the number of concerned frequencies, one problem raises on the validity of modal parameters identification (especially damping ratios) and on the precision of results obtained. This paper presents an OMA method allowing for the evaluation of uncertainties of modal parameters (frequencies, damping ratios and mode shapes). The method is based on the vector autoregressive model (VAR) for multiple numbers of measured channels. It is seen that the uncertainty of modal parameters decreases with higher model orders; the calculation of uncertainty allows also for the construction of an objective criterion for the selection of computing model order, based on a threshold of confidence interval. The derivation confirms also that the identification of natural frequencies deal with a smaller uncertainty compared with the damping ratio estimation, and hence it can be conducted with a lower computing model order. Simulations and real tests on a steel plate show the feasibility and the effectiveness of the developed OMA method.
\end{abstract}

Keywords: Operational modal analysis, vector autoregressive model, model order, uncertainty, noise rate.

\section{Introduction}

Operational modal analysis (OMA) is a useful technique to characterize vibratory properties of machines during industrial applications and to monitor the structural integrity without stopping the normal operation of production. Contrary to usual modal testing techniques, OMA is conducted "in-situ" from only the vibration output signal without knowing any excitation. However, this technique generates more results than necessary. Consequently, a doubt may subsist on the validity and precision of any obtained result, especially in noisy environments. The objective of this paper is to evaluate the uncertainty of any identified modal parameters by using the vector autoregressive model. Moreover, as it is found that uncertainty decreases at higher model orders, a criterion based on an uncertainty threshold is proposed for the selection of computing model order. 


\section{Operational modal analysis by the vector autoregressive model}

In operational modal analysis, the excitation is assumed arbitrary and can be simulated by a Gaussian white noise. Considering a time signal of $d$ synchronized channels acquired at sampling time $T_{\mathrm{s}}$, the data can be modeled by a $p^{\text {th }}$ order vector autoregressive model, Vu et al. [1]:

$$
\mathbf{y}(t)=\boldsymbol{\Lambda} \mathbf{z}(t)+\mathbf{e}(t)
$$

where:

$\boldsymbol{\Lambda}=\left[\begin{array}{llll}-\mathbf{A}_{1} & -\mathbf{A}_{2} & \ldots & -\mathbf{A}_{p}\end{array}\right]$ (size $\left.d \times d p\right)$ is the global model parameters matrix;

$\mathbf{A}_{i}$ (size $\left.d \times d\right)$ is the regression coefficient matrix relates $\mathbf{y}(t-i)$ to $\mathbf{y}(t)$;

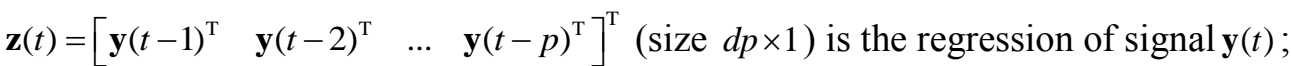

$\mathbf{y}(t-i)($ size $d \times 1 ; i=1: p)$ is the delay signal of a time $i \times T_{\mathrm{s}}$;

$\mathbf{e}(t)$ (size $d \times 1)$ is the model error.

The ambient noise may be assumed as a Gaussian white noise. The least squares estimation method is applicable to estimate the model parameters. With $N$ samples available from $\mathbf{y}(k)$ à $\mathbf{y}(k+N-1)$ $(k>p, N>d p+d)$, the global model parameters matrix $\mathbf{\Lambda}$, the covariance matrix of the deterministic part $\hat{\mathbf{D}}$ et de error part $\hat{\mathbf{E}}$ (both of size $d \times d$ ) can be estimated as follows:

$$
\Lambda=\left(\mathbf{R}_{12}^{\mathrm{T}} \mathbf{R}_{11}\right) \cdot\left(\mathbf{R}_{11}^{\mathrm{T}} \mathbf{R}_{11}\right)^{-1}=\left(\mathbf{R}_{11}^{-1} \mathbf{R}_{12}\right)^{\mathrm{T}}
$$

$$
\hat{\mathbf{D}}=\frac{1}{N} \mathbf{R}_{12}^{\mathrm{T}} \mathbf{R}_{12}
$$

$$
\hat{\mathbf{E}}=\frac{1}{N} \mathbf{R}_{22}^{\mathrm{T}} \mathbf{R}_{22}
$$

In these formulas, $\mathbf{R}_{11}$ (size $d p \times d p$ ), $\mathbf{R}_{12}\left(\operatorname{size} d p \times d\right.$ ) et $\mathbf{R}_{22}$ (size $d \times d$ ) are sub-matrices of the up-diagonal matrix $\mathbf{R}$ (size $N \times d p+d$ ) which is derived from the data matrix par the $\mathrm{QR}$ factorization:

$$
\mathbf{K}=\mathbf{Q} \times \mathbf{R}
$$

where $\mathbf{Q}($ size $N \times N)$ is the orthogonal matrix $\left(\mathbf{Q} \times \mathbf{Q}^{\mathrm{T}}=\mathbf{I}\right)$ and $\mathbf{R}$ has the form as follow:

$$
\mathbf{R}=\left[\begin{array}{cc}
\mathbf{R}_{11} & \mathbf{R}_{12} \\
\mathbf{0} & \mathbf{R}_{22} \\
\mathbf{0} & \mathbf{0}
\end{array}\right]
$$

The data matrix $\mathbf{K}($ size $N \times d p+d)$ is constructed from $N$ successive samples:

$$
\mathbf{K}=\left[\begin{array}{cc}
\mathbf{z}(t)^{\mathrm{T}} & \mathbf{y}(t)^{\mathrm{T}} \\
\mathbf{z}(t+1)^{\mathrm{T}} & \mathbf{y}(t+1)^{\mathrm{T}} \\
\cdots & \cdots \\
\mathbf{z}(t+N-1)^{\mathrm{T}} & \mathbf{y}(t+N-1)^{\mathrm{T}}
\end{array}\right]
$$

Once the model parameters matrix is estimated, the modal parameters are calculated by the eigendecomposition of the state matrix $\Pi$ (size $d p \times d p$ ), Vu et al. [1]:

$$
\boldsymbol{\Pi}=\left[\begin{array}{ccccc}
-\mathbf{A}_{1} & -\mathbf{A}_{2} & \ldots & -\mathbf{A}_{p-1} & -\mathbf{A}_{p} \\
\mathbf{I} & \mathbf{0} & \ldots & \mathbf{0} & \mathbf{0} \\
\mathbf{0} & \mathbf{I} & \ldots & \mathbf{0} & \mathbf{0} \\
\ldots & \ldots & \ldots & \ldots & \ldots \\
\mathbf{0} & \mathbf{0} & \ldots & \mathbf{I} & \mathbf{0}
\end{array}\right]
$$

$$
\boldsymbol{\Pi}=\mathbf{L}\left[\begin{array}{cccc}
\lambda_{1} & 0 & 0 & 0 \\
0 & \lambda_{2} & 0 & 0 \\
0 & 0 & \ddots & \vdots \\
0 & 0 & \ldots & \lambda_{d p}
\end{array}\right] \mathbf{L}^{-1}
$$

where $\lambda_{i}$ is the $i^{\text {th }}$ discrete eigenvalue and $\mathbf{L}$ (size $d p \times d p$ ) is the matrix of eigenvectors: 
$\mathbf{L}=\left[\begin{array}{rrrr}\lambda_{1}^{p-1} \mathbf{l}_{1} & \lambda_{2}^{p-1} \mathbf{l}_{2} & \ldots & \lambda_{d p}^{p-1} \mathbf{l}_{d p} \\ \vdots & \vdots & \vdots & \vdots \\ \lambda_{1} \mathbf{l}_{1} & \lambda_{2} \mathbf{l}_{2} & \ldots & \lambda_{d p} \mathbf{l}_{d p} \\ \mathbf{l}_{1} & \mathbf{l}_{2} & \ldots & \mathbf{l}_{d p}\end{array}\right] \quad(10) \quad \mathbf{S}=\mathbf{L}^{-1}=\left[\begin{array}{cccc}\mathbf{S}_{11} & \mathbf{S}_{12} & \ldots & \mathbf{S}_{1 p} \\ \mathbf{S}_{21} & \mathbf{S}_{22} & \ldots & \mathbf{S}_{2 p} \\ \vdots & \vdots & \ddots & \vdots \\ \mathbf{S}_{d p 1} & \mathbf{S}_{d p 2} & \ldots & \mathbf{S}_{d p p}\end{array}\right]$

\section{Uncertainties of modal parameters by the VAR model}

In operational modal analysis, the errors either from the data acquisition, the identification process or the parameters calculation can generate uncertainties on the modal parameters. Uncertainties on the non parametrical modal analysis methods have been discussed in Mace et al. [2]. The detail of modal parameter uncertainties can be found in Pintelon et al. [3] which showed the calculation of the uncertainties from the covariance matrix by differentiation. It is seen that almost the available calculations of modal parameters use the subspace modal analysis methods, Reynders et al. [4]. With the ARMA time series models, Andersen et al. [5] presented two procedures for calculating uncertainties of modal parameters when the latter are identified by the prediction error method (PEM). Uncertainties of model parameters can also be computed in parallel with the model estimation by the least squares method, Neumaier et al. [6].

In this paper, the computation of modal parameters uncertainties is derived and evaluated with respect to model order and the noise rate. Consider a real scalar function of the model parameters $h=h(\boldsymbol{\Lambda})$; this scalar can be a parameter of the model itself, a natural frequency, a damping ratio or a mode shape component. Interval confidence $\alpha / 100$ of this scalar function can be constructed from the t-distribution of the ratio $t=\frac{h_{ \pm}}{\hat{\sigma}_{h}}$, where $h_{ \pm}=\hat{h}-h$ is the error bound, $\hat{\sigma}_{h}^{2}=\operatorname{Cov}(h(\hat{\mathbf{\Lambda}}))$ is the estimated variance and $N-d^{2} p$ is the number of degrees of freedom. The confidence interval is then calculated, Neumaier et al. [6]:

$$
\hat{h}_{ \pm}=t\left(N-d^{2} p,(1+\alpha / 100) / 2\right) \hat{\sigma}_{h}
$$

The covariance $\operatorname{Cov}(h(\hat{\mathbf{\Lambda}}))$ of the function $h=h(\mathbf{\Lambda})$ can be derived and linearized from the truncated Taylor series. This insures that the covariance matrix is positive semi definite, [6]:

$$
\operatorname{Cov}(h(\hat{\mathbf{\Lambda}}))=\left(\frac{\partial h(\boldsymbol{\Lambda})}{\partial \boldsymbol{\Lambda}}\right)^{T} \operatorname{Cov}(\hat{\mathbf{\Lambda}})\left(\frac{\partial h(\boldsymbol{\Lambda})}{\partial \boldsymbol{\Lambda}}\right)
$$

where the covariance matrix of the least squares estimated model parameters $\operatorname{Cov}(\hat{\mathbf{\Lambda}})\left(\operatorname{size} d^{2} p \times d^{2} p\right)$ is a Kronecker product of the noise estimated variance $\hat{\mathbf{E}}$ (size $d \times d$ ) and the estimation moment matrix $\mathbf{U}$ (size $d p \times d p),[6]:$

$$
\operatorname{Cov}(\hat{\mathbf{\Lambda}})=\mathbf{U}^{-1} \otimes \hat{\mathbf{E}}=\left(\mathbf{R}_{11}^{\mathrm{T}} \mathbf{R}_{11}\right)^{-1} \otimes\left(\mathbf{R}_{22}^{\mathrm{T}} \mathbf{R}_{22}\right)
$$

The calculation of scalar uncertainties falls down to the calculation of its derivative with respect to the model parameters $\dot{h}=\frac{\partial h(\boldsymbol{\Lambda})}{\partial \boldsymbol{\Lambda}}$. Let's start with an eigenvalue, the derivative of the $i^{\text {th }}$ discrete eigenvalue can be computed as given in [6]:

$$
\dot{u}_{i}=\left(\mathbf{L}^{-1} \dot{\mathbf{H}} \mathbf{L}\right)_{i, i}
$$

So the derivative of the $i^{\text {th }}$ continuous eigenvalue is:

$$
\dot{\lambda}_{i}=\frac{\dot{u}_{i}}{u_{i} T_{\mathrm{s}}}=\frac{\left(\mathbf{L}^{-1} \dot{\mathbf{M}} \mathbf{L}\right)_{i, i}}{u_{i} T_{\mathrm{s}}}
$$

From the continuous eigenvalue, the corresponding natural frequency and its derivative can be computed:

$$
f_{i}=\frac{\left|\lambda_{i}\right|}{2 \pi}=\frac{\sqrt{\operatorname{Re}^{2} \lambda_{i}+\operatorname{Im}^{2} \lambda_{i}}}{2 \pi} ; \dot{f}_{i}=\frac{\operatorname{Re} \lambda_{i} \operatorname{Re} \dot{\lambda}_{i}+\operatorname{Im} \lambda_{i} \operatorname{Im} \dot{\lambda}_{i}}{4 \pi^{2} f_{i}}
$$

Also the damping ratio and its derivative of the same mode are: 


$$
\zeta_{i}=-\frac{\operatorname{Re} \lambda_{i}}{\left|\lambda_{i}\right|} ; \dot{\zeta}_{i}=\zeta_{i}\left(\frac{\operatorname{Re} \dot{\lambda}_{i}}{\operatorname{Re} \lambda_{i}}+\frac{\dot{f}_{i}}{f_{i}}\right)
$$

By a simple transformation, we can have the relationship between the derivatives of the natural frequency and of the damping ratio of the same mode as follows. It can be noticed that, at the same model order, the covariance and by consequence, the confidence interval of the damping ratio is greater than the one of the natural frequency:

$$
\frac{\dot{\zeta}_{i}}{\zeta_{i}}=\frac{\dot{f}_{i}}{f_{i}}+\frac{\operatorname{Re} \dot{\lambda}_{i}}{\operatorname{Re} \lambda_{i}}
$$

The real mode shape matrix $\Theta($ size $d \times d p)$ is deduced from amplitudes and phases of the complex eigenvectors matrix $\boldsymbol{\Psi}$. The derivative of a real mode shape component $\dot{\boldsymbol{\Theta}}_{l, i}$ is computed as follows:

$$
\begin{gathered}
\left(\boldsymbol{\Theta}_{l, i}\right)^{2}=\left(\boldsymbol{\Psi}_{l, i}\right)^{2}=\operatorname{Re}^{2}\left(\mathbf{L}_{l, i}\right)+\operatorname{Im}^{2}\left(\mathbf{L}_{l, i}\right) \quad l=1,2, \ldots, d \\
\left|\boldsymbol{\Psi}_{l, i}\right| \dot{\boldsymbol{\Theta}}_{l, i}=\left|\operatorname{Re}\left(\mathbf{L}_{l, i}\right)\right| \operatorname{Re}\left(\dot{\mathbf{L}}_{l, i}\right)+\left|\operatorname{Im}\left(\mathbf{L}_{l, i}\right)\right| \operatorname{Im}\left(\dot{\mathbf{L}}_{l, i}\right)
\end{gathered}
$$

where $\dot{\mathbf{L}}_{l, i}$ is the complex derivative of the element $\mathbf{L}_{l, i}$ of the complex eigenvectors matrix, as given in [6].

\section{Uncertainty evaluation by OMA of a steel plate}

A rectangular steel plate is studied in operational modal analysis. The clamped-free-clamped-free plate is of dimension $500 \times 200 \times 1.9 \mathrm{~mm}$ as shown in Fig. 1 .

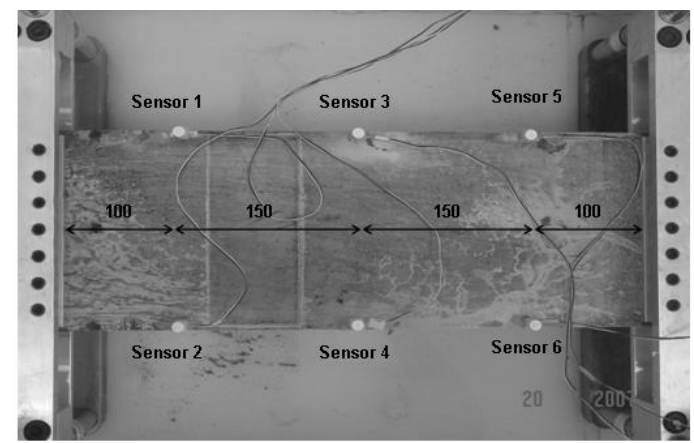

FIG. 1 - Plate configuration with 6 sensors points

\subsection{Numerical simulation of the steel plate}

The plate is firstly simulated by finite element method to produce the time history responses at 6 positions (Fig 1). Numerical vibration data are sampled at $1280 \mathrm{~Hz}$ by the modal superposition method from the 6 first modes with a constant damping ratio of $1 \%$ for each mode.

In order to evaluate the effect of noise rate on the modal parameters uncertainties, different white noise rates are added to the signal at levels of 1\%, 10\% and 100\% RMS. Fig. 2 shows the evolution of the 95\% uncertainty of natural frequencies and damping ratios with respect to the noise rate at the constant model order 20.

a) Frequency $95 \%$ uncertainty vs noise rate

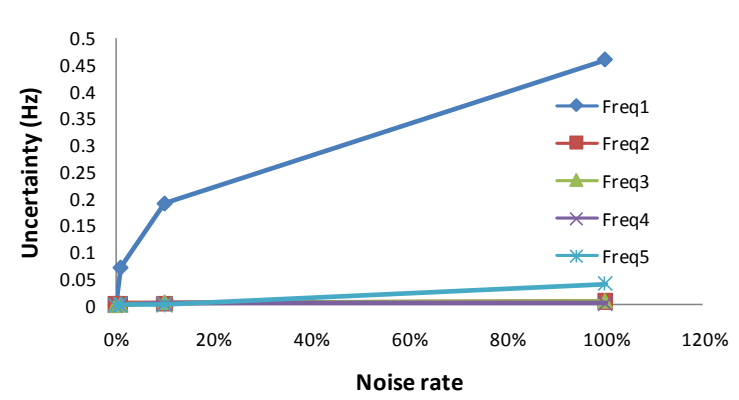

b) Damping ratio $95 \%$ uncertainty vs noise rate

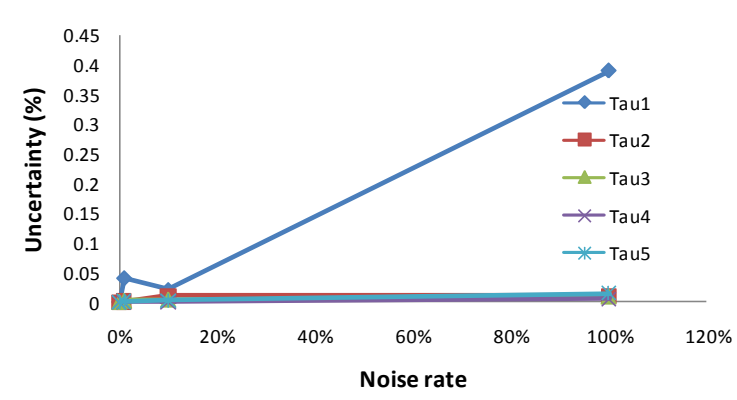

FIG. 2 Modal parameter uncertainties with respect to noise rate 
It can be noticed that the uncertainties of modal parameters increase monotonically with respect to model order but not with a linear rate. At a noise rate less than $10 \%$, the confidence interval of modal parameters can be negligible. At a high noise rate $100 \%$, uncertainty is still small for most of the modes.

\subsection{Experiments on steel plate}

The plate has been excited by impact but with an unmeasured impact force. Time signal data are simultaneously acquired at 6 locations with a sampling frequency of $1280 \mathrm{~Hz}$. The identification of the first mode's natural frequency and damping ratio and their uncertainty with respect to model order is typically shown in Fig.3. The evaluation of 95\% uncertainty of the first 6 natural frequencies and damping ratios with respect to model order is shown in Fig.4.

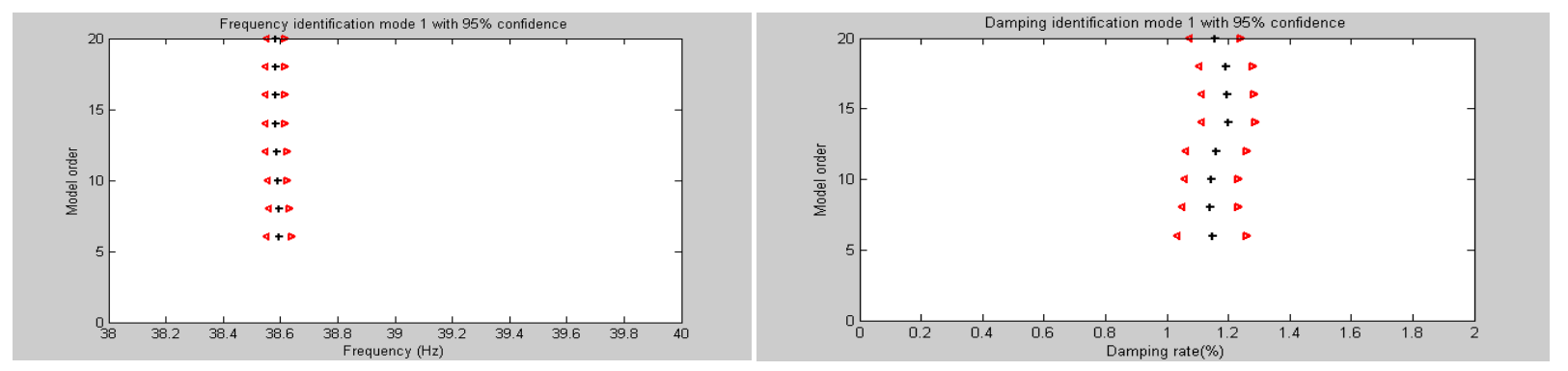

FIG. 3 - Identification of first mode's modal parameters with uncertainties
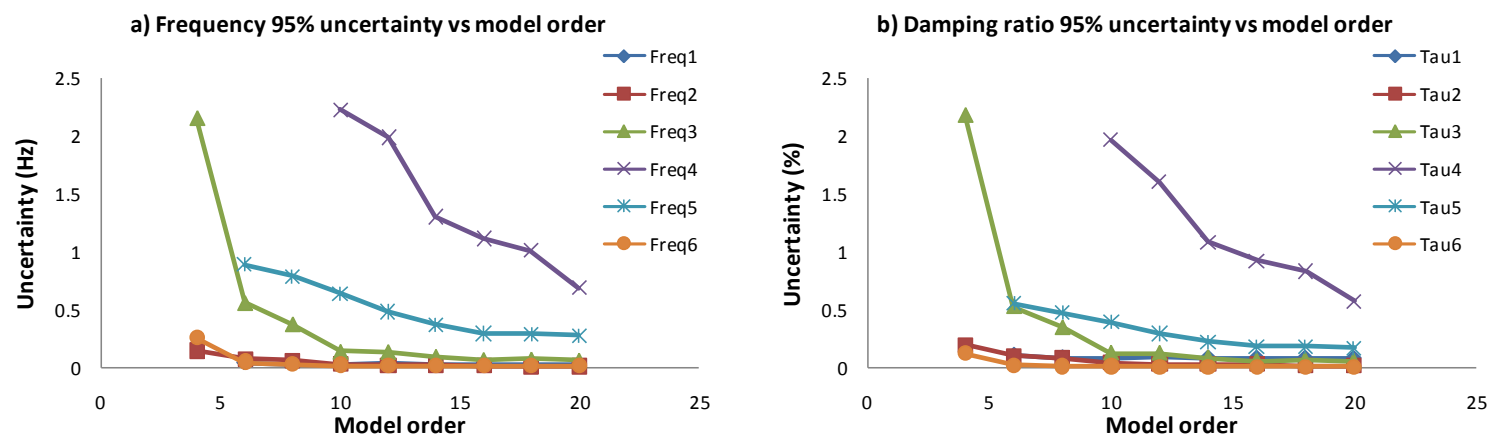

FIG. 4 - Modal parameters uncertainties vs model order

It is found that:

- Uncertainty of all modal parameters decreases when the model order increases, and thus higher the model order is, more the VAR model gives precision in the modal identification.

- At a specific model order, damping ratio's confidence interval is higher than the one of the corresponding natural frequency. This confirms that the identification of natural frequencies is more accurate compared with the identification of damping ratios.

- At the same model order, there are some modes identified with a higher uncertainty compared to other modes (e.g. mode 4 of the plate), either on natural frequency or damping ratio. In order to identify this mode with precision with an acceptable uncertainty, the VAR model needs a higher computing model order. Therefore a threshold on uncertainty of this mode can be defined as a criterion for selecting the computing model order of the whole system.

Fig. 5 displays the identification of mode shapes of the first 6 modes with uncertainties at each location (displayed in color scale on the right, zero uncertainty in dark blue, highest uncertainty in dark red). As expected, the uncertainty of clamped boundaries is null. One can also find that the uncertainty is not always the highest at the location of greatest modal amplitude (points 3 and 4 on center line of the plate, in orange color). For all modes, it is found that at the location 5, the uncertainty is highest (dark red) and the modal amplitude is identified with a highest error. This is due to the fact that the uncertainty depends on the instrumentation setup and the data acquisition at each sensor location. However, it can be noticed that that uncertainties of mode shapes are small in amplitude scale. 

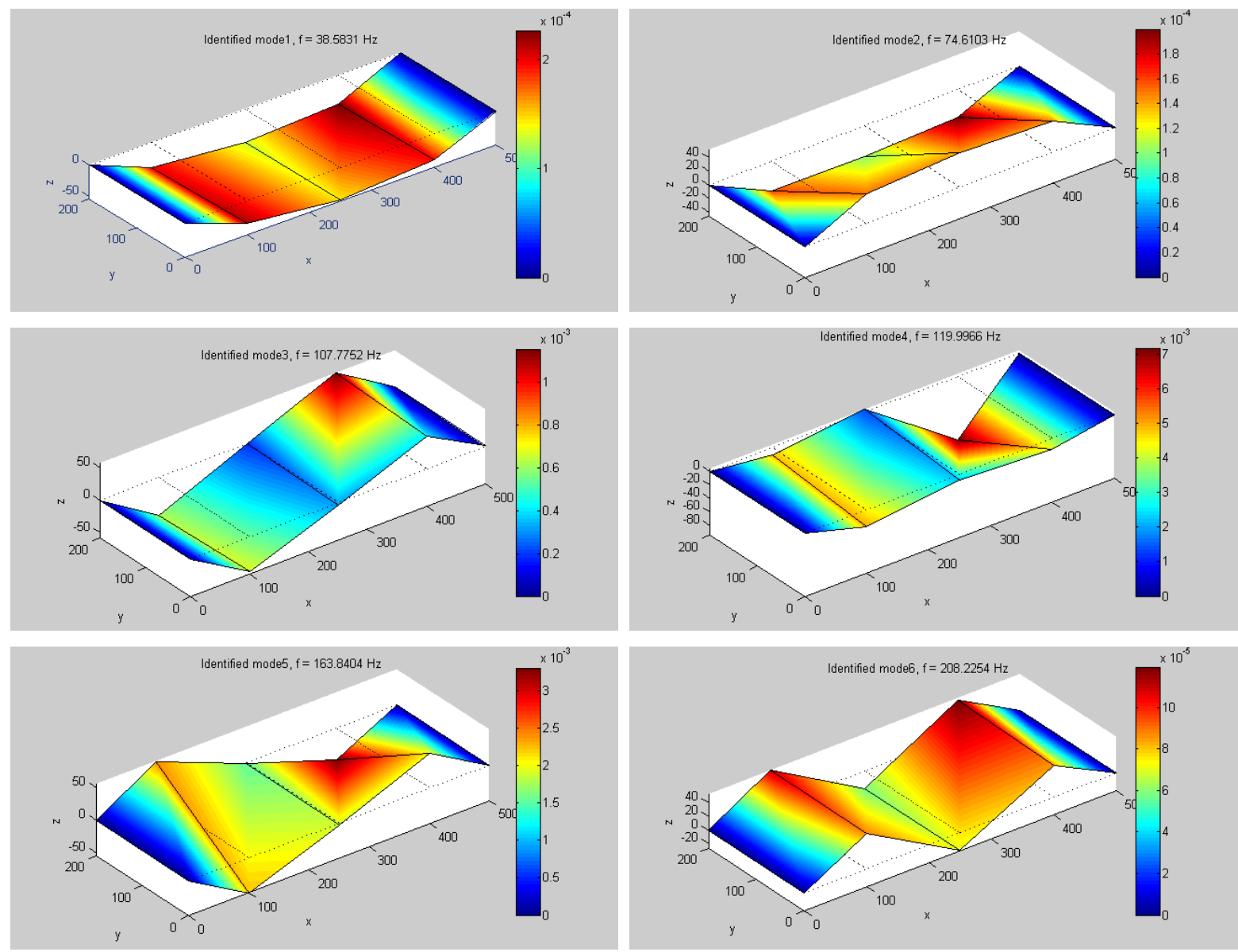

FIG. 5 - Mode shapes identification with uncertainty

\section{Conclusion}

The computation of modal parameters uncertainty in operational modal analysis is presented by using the vector autoregressive model. The ability on estimation of modal parameters uncertainties allows for the establishment of confidence and precision in the identification results on natural frequencies, damping ratios or mode shape components. It can be noticed that uncertainties are higher for the damping ratio estimation than for the natural frequency of the same mode. It is also found that the uncertainty varies from mode to mode in term of natural frequency and damping ratio. It varies also from point to point of the sensors location. A threshold of the uncertainty can be proposed as a criterion for the selection of the computing model order which is a priori unknown in order to identify all the modes with acceptable precision.

\section{References}

[1] Vu V.H., Thomas M., Lakis A.A. and Marcouiller L., (2011), Operational modal analysis by updating autoregressive model, Mechanical systems and signal processing (MSSP), Elsevier, 25(3): 1028-1044.

[2] Mace B. R., K. Worden and G. Manson (2005). Uncertainty in structural dynamics. Journal of Sound and Vibration 288(3): 423-429.

[3] Pintelon R., P. Guillaume and J. Schoukens (2007). Uncertainty calculation in (operational) modal analysis. Mechanical Systems and Signal Processing 21(6): 2359-2373

[4] Reynders E., R. Pintelon, and G. De Roeck, (2008), Uncertainty bounds on modal parameters obtained from stochastic subspace identification. Mechanical Systems and Signal Processing, 22(4): 948-969.

[5] Andersen P. and R. Brinker, (1999), Estimation of Modal Parameters and their Uncertainties, Proceedings of the 17th International Modal Analysis Conference (IMAC), 323-329.

[6] Neumaier A. and Schneider T., (2001), Estimation of parameters and eigenmodes of multivariate autoregressive models. ACM Trans. Math. Softw. 27, pp: 27-57. 